\title{
Title: Cyclic programmed cell death stimulates hormone signaling and root development in Arabidopsis
}

Authors: Wei Xuan ${ }^{1,2,3}$, Leah R. Band ${ }^{4 \#}$, Robert P. Kumpf ${ }^{1,2 \#}$, Daniël Van Damme ${ }^{1,2}$, Boris Parizot $^{1,2}$, Gieljan De Rop ${ }^{1,2}$, Davy Opdenacker ${ }^{1,2}$, Barbara K. Möller ${ }^{1,2}$, Noemi Skorzinski ${ }^{6}$, Maria F. Njo ${ }^{1,2}$, Bert De Rybel ${ }^{1,2,5}$, Dominique Audenaert ${ }^{1,2 \$}$, Moritz K. Nowack ${ }^{1,2}$, Steffen Vanneste $^{1,2}$, Tom Beeckman ${ }^{1,2 *}$

\section{Affiliations:}

${ }^{1}$ Department of Plant Systems Biology, VIB, Technologiepark 927, 9052 Ghent, Belgium

${ }^{2}$ Department of Plant Biotechnology and Bioinformatics, Gent University, Technologiepark 927, 9052 Ghent, Belgium

${ }^{3}$ State Key Laboratory of Crop Genetics and Germplasm Enhancement and MOA Key Laboratory of Plant Nutrition and Fertilization in Lower-Middle Reaches of the Yangtze River, Nanjing Agricultural University, Weigang No.1, Nanjing 210095, PR China

${ }^{4}$ Centre for Plant Integrative Biology, University of Nottingham, Nottingham LE12 5RD, United Kingdom

${ }^{5}$ Laboratory of Biochemistry, Wageningen University, Dreijenlaan 3, 6703HA Wageningen, The Netherlands.

${ }^{6}$ Max Planck Institute for Developmental Biology, Spemannstrasse 35, 72076 Tübingen, Germany

\$Present address: Compound Screening Facility, VIB, Technologiepark 927, 9052 Ghent, Belgium

\#These authors contributed equally to this work.

*Correspondence to: tobee@psb.vib-ugent.be 


\begin{abstract}
The plant root cap, surrounding the very tip of the growing root, perceives and transmits environmental signals to the inner root tissues. In Arabidopsis thaliana, auxin released by the root cap contributes to the regular spacing of lateral organs along the primary root axis. Here, we show that the periodicity of lateral organ induction is driven by recurrent programmed cell death at the most distal edge of the root cap. We suggest that synchronous bursts of cell death in lateral root cap cells release pulses of auxin to surrounding root tissues, establishing the pattern for lateral root formation. The dynamics of root cap turnover may therefore coordinate primary root growth with root branching in order to optimize the uptake of water and nutrients from the soil.
\end{abstract}




\section{Main Text:}

The root cap is the outermost tissue covering the root tip and represents a major rootrhizosphere interaction site (1-3). It is commonly recognized as a protective tissue for the meristematic cells of the root apex and as a sensory organ that perceives environmental signals such as gravity, water and nutrients to direct root growth (4-6). Although it persists during the lifespan of roots, it is subjected to a regeneration process in which new cell layers are continuously produced internally while superficial cell layers are regularly sloughed off. In Arabidopsis, the root cap consists of a central columella and peripheral lateral root cap cells (7). Programmed cell death (PCD) of lateral root cap cells occurs when they reach the onset of the elongation zone (fig. S1A) $(8,9)$. This region is also designated as the oscillation zone, as it displays massive oscillations in gene expression (10). These oscillations periodically define the prebranch sites, which may further develop as lateral roots (10). Root cap-specific conversion of the auxin precursor indole-3-butyric acid (IBA) into indole-3-acetic acid (IAA), creates a local auxin source that is essential for the oscillating transcriptional mechanism which installs the regular spacing of lateral roots $(11,12)$.

Analysis of the transcriptional auxin signalling output reporter DR5rev:VENUS-N7 (13) by stereomicroscopy, revealed a striped DR5 pattern in the most distal lateral root cap cells, a pattern that could also be observed for the root cap expressed early stage programmed cell death (PCD) marker $p P A S P A>>H 2 A-G F P$ (Fig. 1, A and B, fig. S1) (8). In vivo time-lapse imaging of vertically growing roots showed that the most distal stripe of $D R 5$ expression faded out every $\sim 4$ hours (Fig. 1C, fig. S2, A and B, Movie S1). When tracing back the site of origin of lateral root primordia $(\mathrm{n}=96)$ (Fig. 1C), we found that all primordia were initiated at positions where a distal DR5 stripe had vanished. Furthermore, the disappearance of the DR5 signal from the lateral root cap preceeded the DR5:Luciferase maximum in the oscillation zone (fig. S3) and occurred with a similar periodicity (fig. S2B). By rotating the roots by $135^{\circ}$, the orientation of root growth is corrected towards the gravity vector and a bend is formed. During the reorientation, the period of DR5 oscillations in the oscillation zone is transiently shortened, and lateral root formation is stimulated $(10,14-16)$. Likewise, the period between successive losses of DR5 stripes was also shortened from $\sim 4$ to $\sim 2$ hours (fig. S2, C and D, and Movie S2). Altogether, these results show that the disappearance of the DR5 signal from the lateral root cap, 
the DR5 oscillations in the oscillation zone and the formation of lateral root primodium are temporally and spatially interconnected.

The longitudinal extent of the lateral root cap is developmentally restricted by induction of PCD in the most distal lateral root cap cells (8), raising the possibility that the periodic disappearance of the DR5 signal coincides with PCD in the lateral root cap. Consistently, pPASPA3 > > H2A-GFP showed a striped pattern in the lateral root cap (Fig. 1B). Moreover, coexpression of the DR5rev:VENUS-N7 reporter with pPASPA3:NLS-tdTomato revealed overlapping expression in the most distal lateral root cap cells (Fig. 2A, and fig. S4A). Timelapses showed that both signals disappeared synchronously (Fig. 2A, and fig. S4B), with a period of $\sim$ h (fig. S2B), and spatially correlating with sites of new lateral root primordia (Movie S3). Moreover, a $135^{\circ}$ gravistimulation also transiently decreased the periodicity of disappearance of PASPA3 stripes to $\sim 2$ hours (fig. S2D, and Movie S4). Thus, PCD in the lateral root cap is predictive of lateral root formation.

In Arabidopsis, the accurate timing of PCD in the lateral root cap requires the transcription factor SMB $(8,17)$. pSMB:NLS-GFP stripes overlapped with pPASPA3:NLS-tdTomato stripes in the most distal lateral root cap and disappeared $\sim 4$ hours (fig. S2B and S4C). The $s m b-3$ mutant exhibits delayed PCD of the lateral root cap cells $(8,17)$, and as a result, has an increased number of the lateral root cap cells that ectopically extend into the elongation zone (fig. S5, A and B) $(8,17)$. In this mutant, the typical stripe-like pattern of DR5 expression had disappeared (Fig. 2B) while the signal intensity was reduced and extended into the elongation zone (fig. S5, A and C). Moreover, we observed a more variable periodicity of DR5:Luciferase oscillations (fig. S5D), fewer prebranch sites and lateral roots (fig. S5, E to H). Strong activation of SMBGR (10 $\mu \mathrm{M}$ Dexamethasone (Dex)) triggers ectopic formation of tracheary element-like cells and growth arrest of all tissues (17). Over a 2-day treatment with $\leq 1 \mu \mathrm{M}$ Dex, root growth was maintained (fig. S6A) while showing a pronounced and specific PCD in the lateral root cap cells (fig. S6B). Additionally, these roots lacked DR5 stripes (Fig. 2C), DR5:Luciferase oscillations (Fig. 2, D and E, and Movie S5) and the number of pre-branch sites and lateral roots was reduced respectively by $79.4 \%$ and $87.5 \%$ at $0.3 \mu \mathrm{M}$ Dex (Fig. 2F, and fig. S6, A and C). When plants were transferred back to control medium, the newly formed root segment reestablished normal growth with the production of a normal lateral root cap and lateral roots (fig. S6, D to F). In contrast, the part of the root that was formed during Dex treatment remained devoid of lateral 
roots (fig. S6, D and E). These results indicate that the controlled and recurrent PCD of the lateral root cap cells is the driving factor for gene expression oscillations in the oscillation zone and subsequent lateral root induction.

Oscillations are modulated by a local auxin source in the root cap, derived from the auxin precursor IBA $(11,12)$. Moreover, genetic ablation of the lateral root cap cells represssed the capacity to produce extra lateral roots in response to exogenous IBA application in Dex-treated 35S:SMB-GR (fig. S6G). Therefore, we asked whether the auxin response that we observed in the root cap itself could be required for lateral root patterning. We conditionally repressed the auxin response in the lateral root cap cells by activation of a stabilized allele of the auxin response repressor IAA17/AXR3 (pSMB:axr3-1-GR) $(5,18)$. Dex treatment resulted in agravitropic root growth (fig. S7A) and loss of DR5 expression in the lateral root cap cells (fig. S7, B and C), but this did not alter the PCD process (fig. S7D) and did not affect the lateral root number (fig. S7E). Constitutive transactivation of $U A S:$ axr3-1 in the lateral root cap only slightly reduced lateral root formation, whereas transactivation of UAS:axr3-1 in xylem pole pericycle cells blocked lateral root formation (fig. S7F) (19). Therefore a transcriptional auxin response in the lateral root cap itself is not a decisive factor for lateral root patterning.

Alternatively, auxin transport from the root cap to the root proper could be the connecting element for the oscillatory behavior in gene expression in the elongation zone. Consistently, time-lapse analyses of the semi-quantitative auxin input reporter R2D2 revealed a marked increase of auxin levels in epidermal cells, prior to loss of cellular integrity of adjacent lateral root cap cells (Fig. 3A, Movie S6) (20). This suggests that auxin released from lateral root cap cells during a late stage of PCD is efficiently taken up by the abutting epidermal cells. To understand how this could result in auxin signalling in stele cells of the oscillation zone, we adopted an in silico auxin-transport model (21) to simulate the auxin dynamics in the root apex (See Supplementary Information for further details). Simulating the PCD of distal lateral root cap cells, under the assumption that PCD leads to a release of auxin into the surrounding apoplast, generated a transient auxin peak in stele cells in the elongation zone (Fig. 3, B to D, fig. S8, and Movie S7), consistent with the oscillating activation of the DR5:Luciferase. When defects in either auxin uptake or IBA conversion are prescribed, the model fails to predict such a transient increase in stele auxin levels after lateral root cap cell turn-over (Fig. 3, B to D, fig. S8, and Movie S7). Our model suggests that the shootward auxin flux to the oscillation zone requires 
lateral root cap-expressed AUX1 (fig. S9, A and B), while auxin production was predicted to create high auxin levels in the lateral root cap prior to PCD.

Consistent with these simulations, we observed a reduced DR5 signal in the lateral root cap which was correlated with less prebranch sites and lateral roots in ibrlibr3ibr10 and auxl mutants (Fig. 4, A and B). Moreover, PCD in the most distal lateral root cap cells was closely associated with increased auxin in the underlying epidermal cells (Fig. 3A, Movie S6). We further tested the contribution of auxin transport within the lateral root cap by tissue-specific complementation of the auxl mutant. In agreement with model predictions (fig. S9, C to E), transactivation of AUX1 in the root cap rescued the defect in lateral root formation and agravitropic growth of auxl mutants (fig. S9, F and G). Thus, auxin transported within the (lateral) root cap allows the root cap to communicate with the elongation zone for establishing sites for lateral roots to develop. This process ensures that IBA-derived auxin can be transported towards the oscillation zone.

The auxin-transport topologies in our model also include carrier-mediated efflux and apoplastic diffusion. In the presence of influx carriers and auxin production, simulations lacking carrier-mediated efflux failed to generate an auxin transient in the elongation zone, but generated an auxin accumulation in the lateral root cap (Fig. 3, B to D, fig. S8, and Movie S7). In our model diffusion rates were positively correlated with the strenght of the auxin peak in the stele. However, such higher apoplastic diffusion rate could not compensate for a lack in auxin efflux in our simulations (fig. S10). In an attempt to identify the components of this auxin transport machinery, we analysed pin2 and pin2 abcbl abcb19 mutants. Although these mutants are severely defective in shootward auxin transport and gravitropism, similar to auxl (23), they did not show defects in lateral root formation, nor did they have a reduced sensitivity to IBA (fig. S11, A to D), raising the possiblity that this reflux model requires the global features of the PIN and $\mathrm{ABCB}$ localization for directing auxin into the oscillation zone $(26,27)$. We could find further evidence by using three chemically unrelated auxin transport inhibitors 1-NNaphtylPhtalamic Acid (NPA), 2-[4-(diethylamino)-2-hydroxybenzoyl]benzoic acid (BUM), and benzyloxy-IAA (Bz-IAA), that target mainly ABCB-type transporters (NPA and BUM) (22, 23), or generally interfere with AUX1, PIN and ABCB-based auxin transport (Bz-IAA) (24). Consistent with our simulations, treatments with any of these inhibitors preserved the occurrence of PCD in the lateral root cap (fig. S11E), but resulted in ectopic DR5 activity in the lateral root 
cap and epidermis (Fig. 4, C and D, fig. S11, F and G, and Movie S8), as well as impaired DR5:Luciferase oscillations (Movie S9) and lateral root formation (Fig. 4E, and fig. S11, H and I), corroborating the auxin reflux model (25). Although we could not completely resolve the molecular mechanism for auxin efflux at present, our data underscores the necessity of auxin transport in the coordination of PCD in the most distal lateral root cap cells with oscillatory gene expression in the oscillation zone for lateral root spacing (fig. S12).

During the exploration of the soil, root tips sense, through the root cap, the nutrient and water status of the soil they are traversing, as well as obstacles they may encounter $(6,28)$. Transduction of that information may serve to control the periodicity of programmed cell death, thus altering the frequency of lateral root development. In this way root systems may adjust development according to the quality of the soils they are passing through. 


\section{Acknowledgements}

This work was funded by grants from the Interuniversity Attraction Poles Programme (IAP VI/33 and IUAP P7/29 'MARS') initiated by the Belgian Science Policy Office, the Research Foundation-Flanders and Ghent University Special Research Fund to T.B.; from the Netherlands Organization for Scientific Research (NWO VIDI 864.13.001) to B.D.R.. W.X. is supported by a grant from the Chinese Scholarship Council (CSC) with cofunding from Ghent University Special Research Fund, and a grant sponsored by 111 Project (B12009) and Innovative Research Team Development Plan of the Ministry of Education of China (IRT1256), and PAPD in Jiangsu Higher Education Institutions. L.B. is supported by a Leverhulme Trust Early Career Fellowship and a BBSRC New Investigator grant. B.K.M. and R.P.K. are the recipients of Omics@ vib Marie Curie COFUND fellowship, S.V. is a Postdoctoral Fellow of the Research FoundationFlanders. We thank Prof. Malcolm Bennett (University of Nottingham, UK), Prof. Ben Scheres (Wageningen University, the Netherlands), Prof. Philip N. Benfey (Duke University, USA), Prof. Jiří Friml (IST, Austria), Dr. Lucia C. Strader (Washington University, St. Louis, MO, USA), Prof. Markus Geisler (University of Fribourg, Fribourg, Switzerland) and Prof. Niko Geldner (University of Lausanne, Switzerland) for the kind gifts of materials. We thank Matthias Van Durme for the help in cloning. The authors declare that they have no conflicts of interest.

Supplement contains additional data.

\section{Author contributions:}

W.X. and D.V.D. set up the in vivo root imaging system. W.X., R.P.K., G.D.R. and D.O. performed imaging and analysis. L.B. developed the mathematical modelling. W.X., B.D.R., and D.A. collected and analyzed the auxin transport mutants. B.P. and M.F.N. assisted in data analysis and art work. N.S. and M.K.N. provided material for the study. W.X., S.V. and T.B. directed and wrote the manuscript. L.B. and B.K.M. helped with the writing. All authors discussed the results and contributed in the finalization of the manuscript. 


\section{Reference}

1. U. Baetz, E. Martinoia, Trends Plant Sci. 19, 90-98 (2014).

2. A. Driouich, M. L. Follet-Gueye, M. Vicre-Gibouin, M. Hawes, Curr. Opin. Plant Biol. 16, 489-495 (2013).

3. B. Plancot et al., Plant Physiol. 163, 1584-1597 (2013).

4. $\quad$ L. R. Band et al., Proc. Natl. Acad. Sci. USA 109, 4668-4673 (2012).

5. $\quad$ R. Swarup et al., Nat. Cell Biol. 7, 1057-1065 (2005).

6. $\quad$ S. Svistoonoff et al., Nat. Genet. 39, $792-796$ (2007).

7. $\quad$ L. Dolan et al., Development 119, 71-84 (1993).

8. M. Fendrych et al., Curr. Biol. 24, 931-940 (2014).

9. $\quad$ R. P. Kumpf, M. K. Nowack, J. Exp. Bot. 66, 5651-5662 (2015).

10. M. A. Moreno-Risueno et al., Science 329, 1306-1311 (2010).

11. B. De Rybel et al., Nat. Chem. Biol. 8, 798-805 (2012).

12. W. Xuan et al., Curr. Biol. 25, 1381-1388 (2015).

13. M. G. Heisler et al., Curr. Biol. 15, 1899-1911 (2005).

14. M. Lucas, C. Godin, C. Jay-Allemand, L. Laplaze, J. Exp. Bot. 59, 55-66 (2008).

15. F. A. Ditengou et al., Proc. Natl. Acad. Sci. USA 105, 18818-18823 (2008).

16. J. M. Van Norman, W. Xuan, T. Beeckman, P. N. Benfey, Development 140, 4301-4310 (2013).

17. T. Bennett et al., Plant cell 22, 640-654 (2010).

18. D. Rouse et al., Science 279, 1371-1373 (1998)

19. I. De Smet et al., Development 134, 681-690 (2007).

20. C. Y. Liao et al., Nat. Methods 12, 207-210 (2015).

21. L. R. Band et al., Plant cell 26, 862-875 (2014).

22. J. Y. Kim et al., J. Biol. Chem. 285, 23309-23317 (2010).

23. S. Henrichs et al., EMBO J. 31, 2965-2980 (2012).

24. E. Tsuda et al., J. Biol. Chem. 286, 2354-2364 (2011).

25. I. Blilou et al., Nature 433, 39-44 (2005).

26. V. A. Grieneisen, J. Xu, A. F. Maree, P. Hogeweg, B. Scheres, Nature 449, 1008-1013 (2007).

27. M. Laskowski et al., PLoS Biol. 6, e307 (2008).

28. Y. Bao et al., Proc. Natl. Acad. Sci. USA 111, 9319-9324 (2014). 


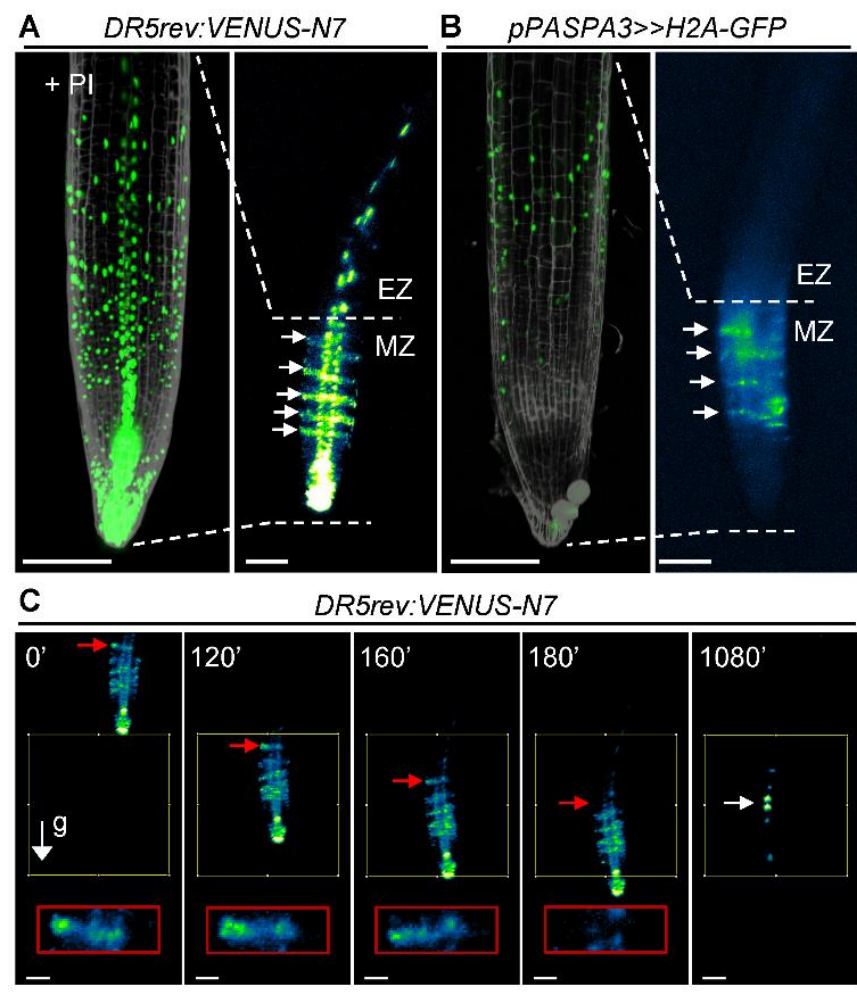

Fig. 1. Periodic disappearance of lateral root cap-DR5 expression correlates with the sites of lateral root initiation. (A and B) 3D-projection of confocal z-stacks (PI stained, left panels) and macroview stereo microscope images (right panels) of respectively, (A) the auxin responsive marker DR5rev:VENUS-N7 expression and, (B) the PCD marker $p P A S P A 3>>H 2 A-G F P$ in seedling root tips. EZ, elongation zone; MZ, meristematic zone. (C) Time lapse imaging of growing DR5rev:VENUS-N7 roots over a growth period of 18 hours. Red arrows: the disappearance of a DR5 stripe in the lateral root cap. White arrow: a lateral root initiation site at the site where the DR5 stripe disappeared previously. Insets show the gradual reduction of DR5 signal intensity in the most distal lateral root cap cells highlighted by the arrowhead during the first $3 \mathrm{~h}$. ' $\mathrm{g}$ ' and associated arrow indicate the gravitational vector. Scale bars, $100 \mu \mathrm{m}$. 
A

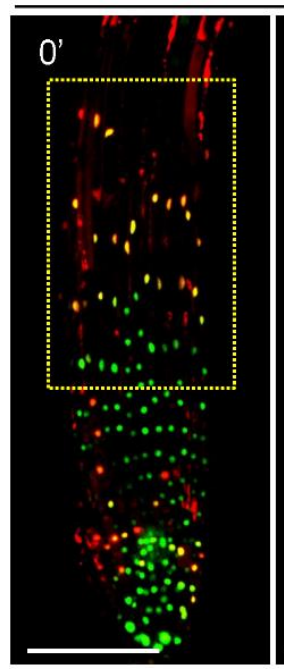

DR5rev:VENUS-N7 X PPASPA3:NLS-tdTOMATO
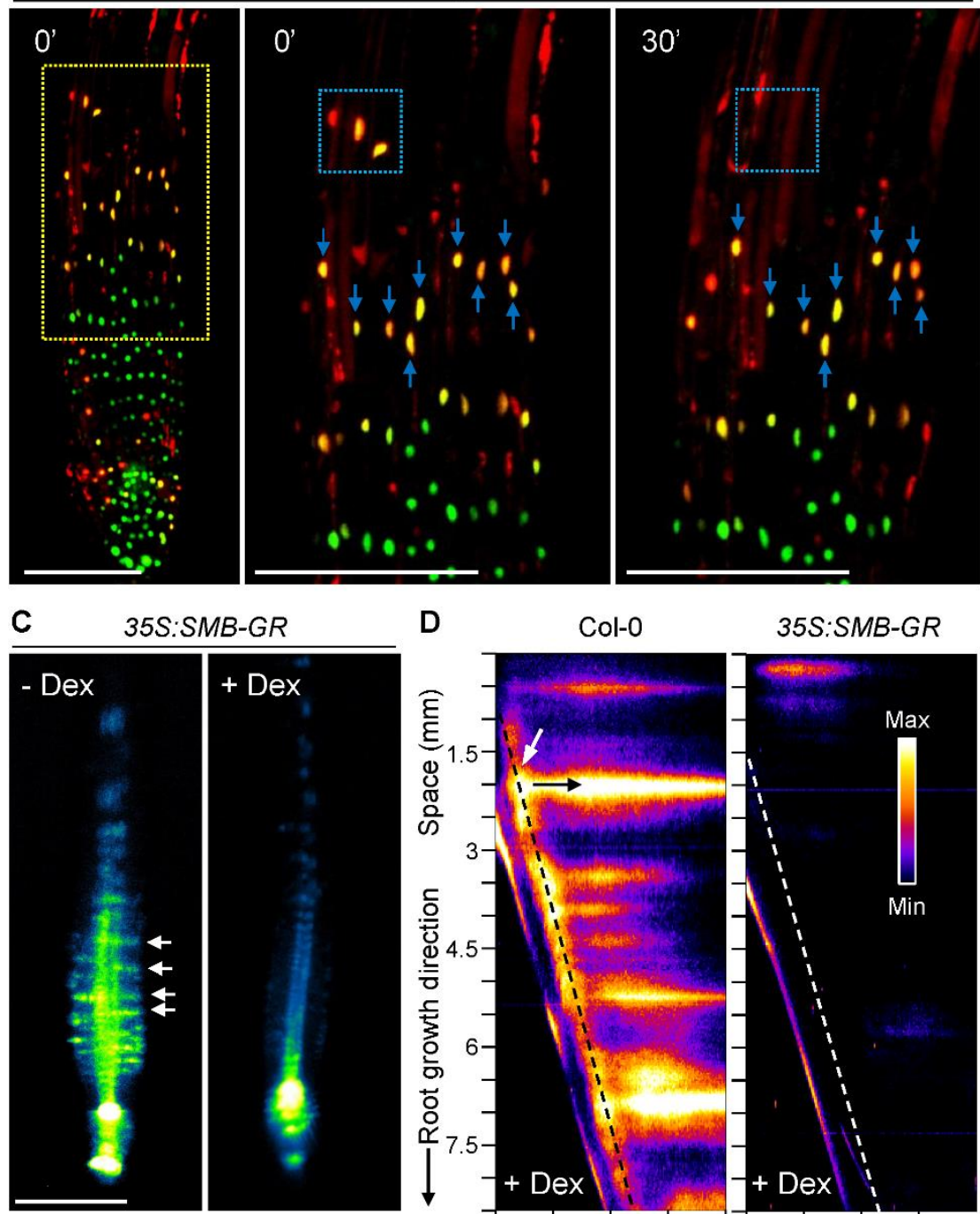

D

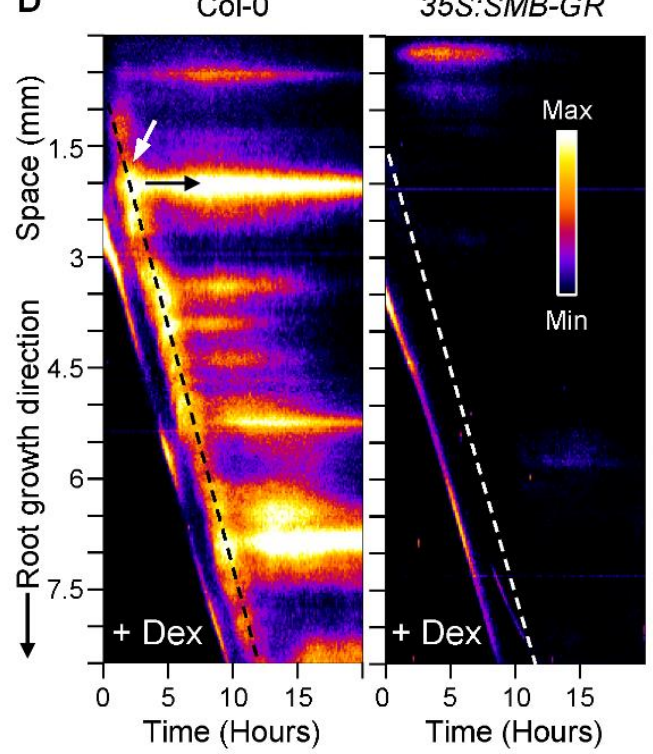

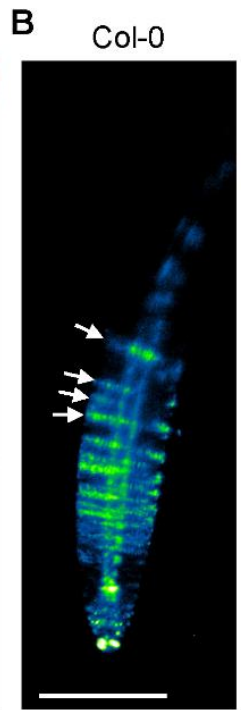

smb-3

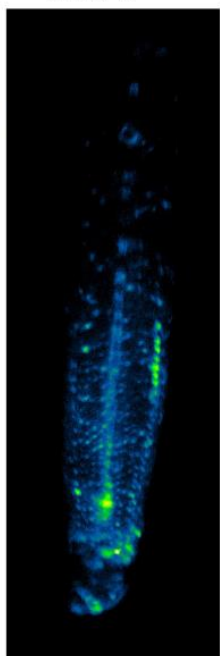

$\mathrm{E}$
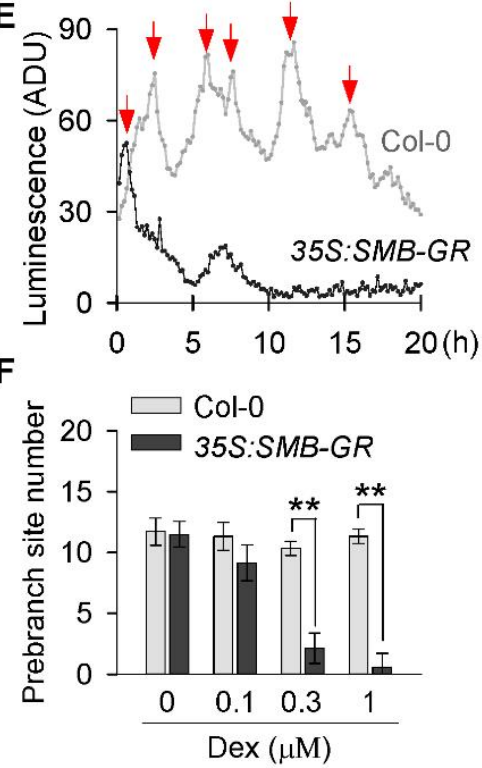

Fig. 2. Disappearance of $D R 5$ expression in the lateral root cap is triggered by PCD. (A) 3D-projection of confocal z-stacks of a DR5rev:VENUS-N7 (green) and pPASPA3:NLStdTomato (red) expressing transgenic seedling root tip imaged over time. Yellow indicates coexpression of both makers. The right panels represent a close-up of the yellow boxed region in the left panel over a 30 min time-frame. Blue inset boxes indicate a set of nuclei expressing both markers that undergo PCD between the 0' and 30' time points. Blue arrows indicate a set of lateral root cap nuclei for positional reference. (B and $\mathbf{C}$ ) Macroview stereo microscopic view of DR5rev:VENUS-N7 expression in root tips of 3-day-old (B) Col-0 and smb-3 seedlings and (C) 35S:SMB-GR seedlings treated for $24 \mathrm{~h}$ with or without $1 \mu \mathrm{M}$ Dex. White arrows: DR5 stripes in the lateral root cap. (D) Kymograph representing DR5:Luciferase expression in Col-0 and 
35S:SMB-GR transgenic seedlings over $20 \mathrm{~h}$ treatment with $1 \mu \mathrm{M}$ Dex. Dashed lines highlight the position of the oscillation zone. White arrow: onset of DR5 maximum; black arrow: prebranch site indicated by persistent DR5 signal. (E) Quantification of DR5:Luciferase luminescence over time measured along the dashed lines shown in (D). Red arrows: oscillation peaks. (F) Quantification of prebranch site number in 5-day-old 35S:SMB-GR seedlings treated with Dex since day 3 after germination $(n>10)$. The prebranch sites from the newly grown primary root after transfer were counted. ${ }^{* *} P<0.01$ indicates significant difference by Student's $t$ test (n > 30). Data are means \pm s.d. Scale bars, $200 \mu \mathrm{m}$. 

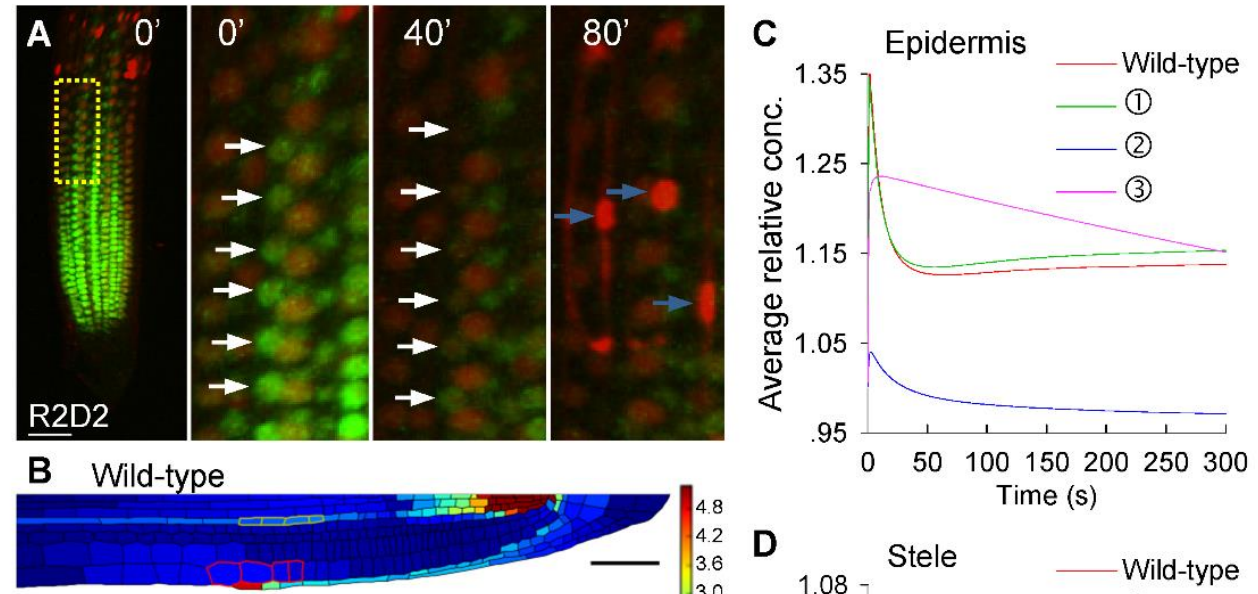

(1) Defect in IBA-to-IAA conversion

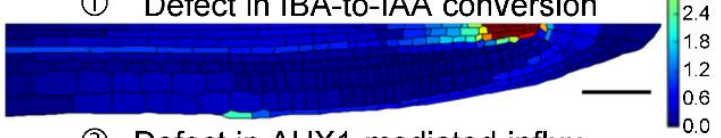

(2) Defect in AUX1-mediated influx

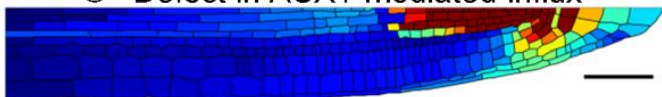

(3) Defect in carrier-mediated efflux
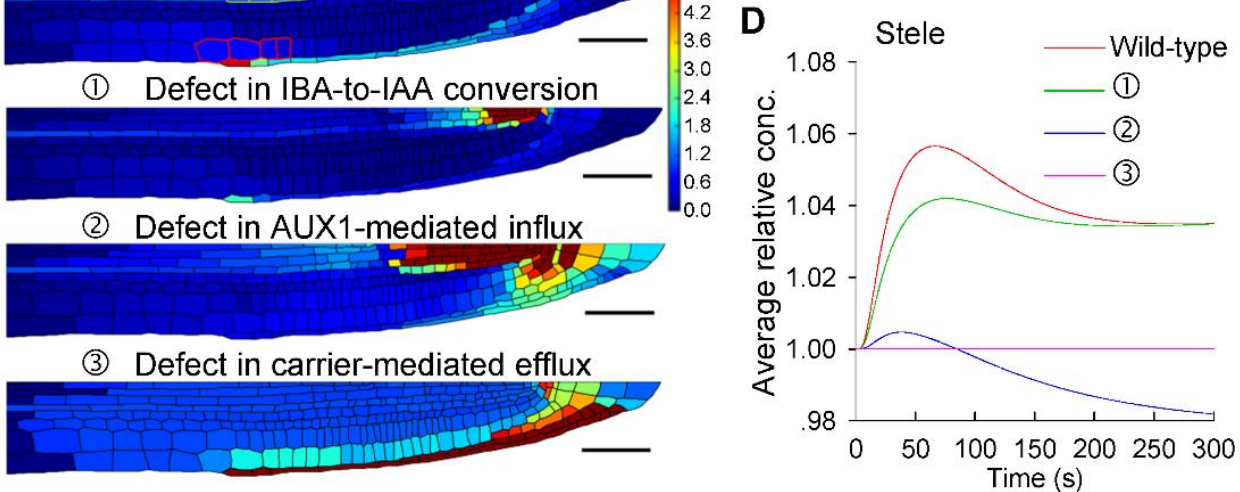

Fig. 3. Predicted auxin distributions and dynamics in the root tip. (A) 3D-projection of confocal z-stacks of a R2D2 root tip, co-stained with PI, imaged over time. The right panels represent a close-up of the yellow boxed region in the left panel over an 80 min time-frame. White arrows indicate the disappearance of YFP signals in epidermal cells, and blue arrows indicate the appearance of PI-stained nuclei in lateral root cap cells, as a late stage PCD marker, at positions where R2D2 signals in the epidermis changed at earlier time-points (see also Movie S6). (B) Predicted steady-state distribution of auxin within a 3D axisymmetric multicellular geometry, for wild type, and in the situations with defects in IBA-to-IAA conversion, AUX1mediated influx and polar carrier-mediated efflux respectively. Auxin concentrations are colour coded according to the rainbow scale on the right-hand side. (C and D) Predicted auxin dynamics in the (C) epidermal cells and (D) the stele cells underlying the most distal LRC cells after PCD. Results show the auxin concentrations relative to that at $\mathrm{t}=0$, taken as an average of the four epidermal cells (marker in red) or stele cells (marked in yellow) highlighted in (B). Scale bars, $50 \mu \mathrm{m}$. 


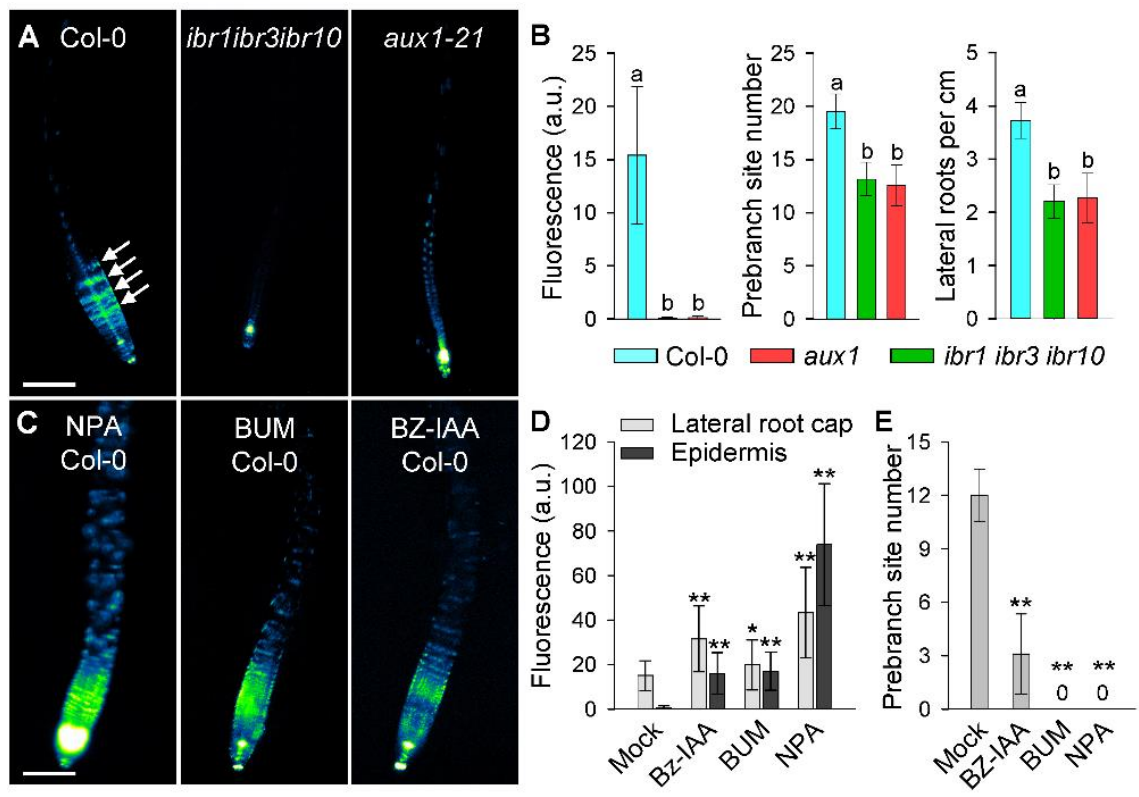

Fig. 4. Auxin flux carriers facilitate auxin transport from the lateral root cap into the oscillation zone. (A) DR5rev:VENUS-N7 expression in 3-day-old Col-0, ibrlibr3ibr10 mutant in control conditions, in macroview stereomicroscope images. White arrows indicate the nuclear DR5 signal in lateral root cap cells. (B) Quantification of DR5rev:VENUS-N7 signal in lateral root cap, prebranch site number per root, and lateral root density in Col-0, ibrlibr3ibr10, and auxl seedlings $(P<0.05$ by one way ANOVA and Tukey's test as post hoc analysis, $\mathrm{n}>10)$. $(\mathbf{C}$ and D) DR5rev:VENUS-N7 expression and quantification in 3-day-old Col-0 germinated on 10 $\mu \mathrm{M}$ NPA, $0.3 \mu \mathrm{M}$ BUM, and $3 \mu \mathrm{M}$ BZ-IAA expanded DR5 expression in WT compared to Col-0 in (A). (E) Quantification of prebranch site number in 5-day-old DR5:Luciferase seedlings treated for 2 days with $10 \mu \mathrm{M}$ NPA, $0.3 \mu \mathrm{M}$ BUM, and $3 \mu \mathrm{M}$ BZ-IAA. The prebranch sites formed in the newly grown primary root after transfer were measured. $* P<0.05$ and $* * P<0.01$ compared to Mock-treatment Col-0 in (A) by Student's $t$ test (n > 30). Data are means \pm s.d. Scale bars, $200 \mu \mathrm{m}$. 